\title{
Vomiting in a 22-day-old firstborn male
}

\author{
Brian T. Kloss • Shaheena R. Patierno
}

Received: 18 February 2010 / Accepted: 7 April 2010 /Published online: 13 July 2010

(C) Springer-Verlag London Ltd 2010

A 22-day-old healthy appearing male infant presented with persistent vomiting after feeding with onset shortly after birth. This is the father's second child and the mother's first. Switching formulas had been unsuccessful. Abdominal X-ray (AXR) revealed a dilated air-filled stomach with a nonspecific bowel gas pattern (Fig. 1). Ultrasound (US) demonstrated a thick and elongated pylorus, confirming the diagnosis (Figs. 2 and 3).

Pyloric stenosis is an important cause of gastric outlet obstruction and vomiting in infants. Peak incidence occurs between 2 and 5 weeks of age with an incidence of 1-8 per 1,000 live births. Male infants are predominantly affected 4:1 over female infants [1]. There is a familial component, with sons of affected fathers carrying a $5 \%$ risk of pyloric stenosis [2].

The cardinal feature of this disorder is projectile, nonbilious vomiting that occurs anywhere from the end of feeding up to $30 \mathrm{~min}$ after. Afterwards the infant exhibits a desire to feed more. Persistent emesis results in hypokalemic hypochloremic metabolic alkalosis with dehydration. Palpation of an olive-shaped, muscular, mobile, and nontender mass in the left upper quadrant is pathognomonic of this condition. During feeding, gastric peristaltic waves can

All work was completed at SUNY Upstate Medical University. Institutional Review Board exemption for a case report was obtained. Brian T. Kloss, DO, JD, PA-C has ultimate control of the submitted abstract and case report/clinical image.

\footnotetext{
B. T. Kloss $(\bowtie) \cdot$ S. R. Patierno

Department of Emergency Medicine,

SUNY Upstate Medical University,

550 East Genesee Street,

Syracuse, NY 13202, USA

e-mail: klossb@upstate.edu

S. R. Patierno

e-mail: Bielmans@upstate.edu
}

sometimes be observed from left to right upper abdominal quadrants.

Radiographic signs of pyloric stenosis include gastric distension with air, a maximal gastric diameter of $7 \mathrm{~cm}$ or greater, and thick walled gastric antrum [3]. Abdominal US confirms the diagnosis by illustrating the increased length and thickness of the pyloric muscle.

Before surgical treatment, correction of dehydration and serum electrolytes is necessary. Definitive therapy is pyloromyotomy with an excellent outcome [4].

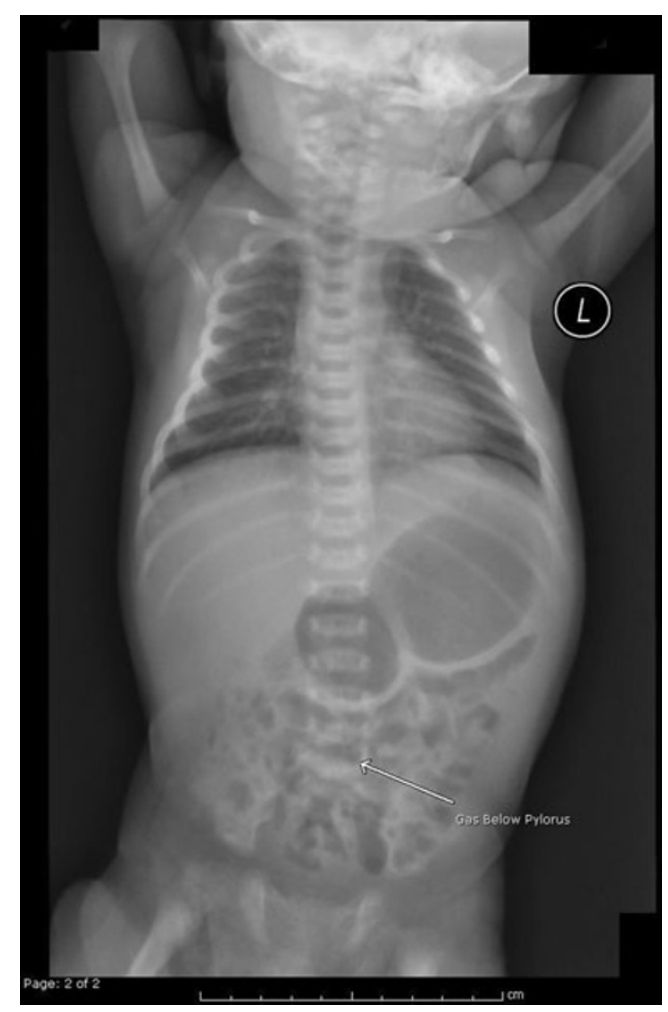

Fig. 1 AXR showing a dilated and air-filled stomach 


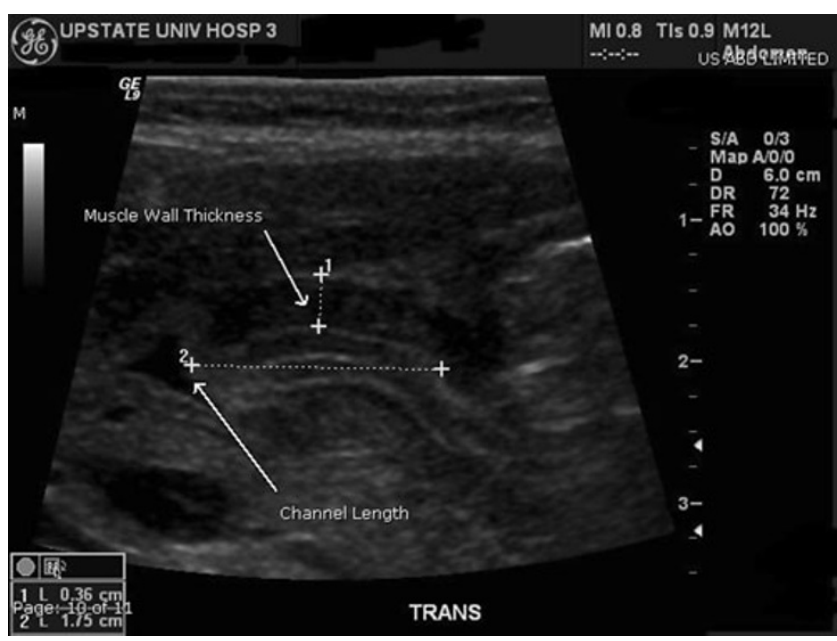

Fig. 2 US showing an elongated and thickened pylorus

\section{References}

1. Liao Z, Li Z, Zhang W et al (2007) Education and imaging. Gastrointestinal: infantile hypertrophic pyloric stenosis. J Gastroenterol Hepatol 22(10):1692

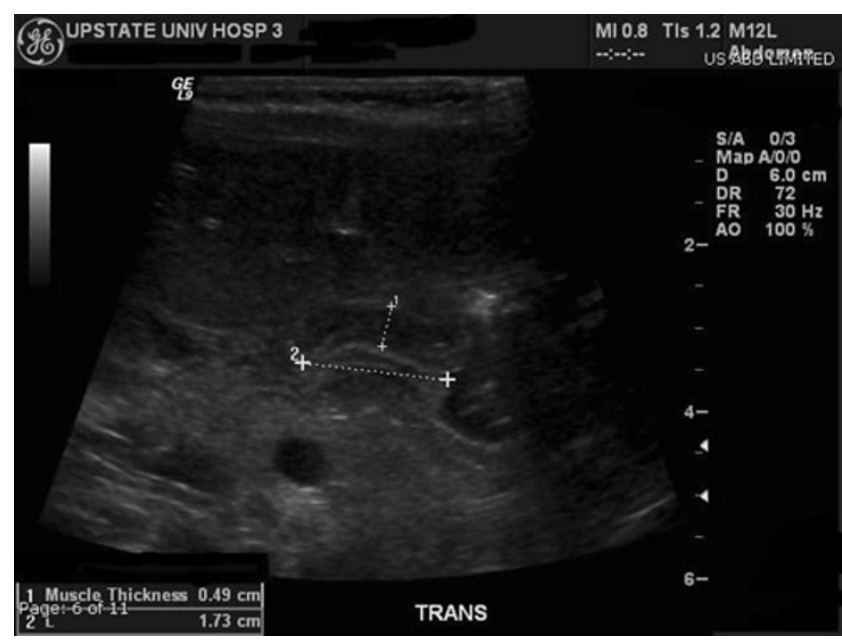

Fig. 3 US view from a slightly different angle confirms the measurements

2. Hernanz-Schulman M (2009) Pyloric stenosis: role of imaging. Pediatr Radiol 39(Suppl 2):S134-S139

3. Haller JO, Cohen HL (1986) Hypertrophic pyloric stenosis: diagnosis using US. Radiology 161(2):335-339

4. Aspelund G, Langer JC (2007) Current management of hypertrophic pyloric stenosis. Semin Pediatr Surg 16(1):27-33 